\title{
DATA PROCESSING OF MEASURED SURFACE TEMPERATURES OF CONTINUOUSLY CAST BILLETS AND BLOOMS TO VERIFY THE NUMERICAL SOLIDIFICATION MODEL
}

\author{
${ }^{1}$ René PYSZKO, ${ }^{1}$ Miroslav PŘíHODA, ${ }^{1}$ Mário MACHU゚, ${ }^{2} Z$ deněk FRANĚK \\ 'VSB - Technical University of Ostrava, Ostrava, Czech Republic, EU, \\ rene.pyszko@vsb.cz, miroslav.prihoda@vsb.cz, mario.machu@vsb.cz \\ ${ }^{2}$ Silesian University in Opava, School of Business Administration in Karvina, Karvina, Czech Republic, EU, \\ franek@opf.slu.cz
}

https://doi.org/10.37904/metal.2020.3447

\begin{abstract}
Numerical models of solidification and cooling of continuously cast billets or blooms are used both in research and in operational conditions to predict solid shell thickness, metallurgical length, solidification rate etc. The numerical model must be verified according to real values of quantities. Although several different quantities can be used to verify the model, most often the models are verified by comparing the calculated and measured surface temperatures of the strand in the secondary and tertiary cooling zones.

The casting process is influenced by a number of known and hidden parameters, often time-varying, which are reflected in the measured surface temperatures, but which cannot be incorporated into the model due to a lack of information to define the exact boundary conditions. For the purposes of model verification, it is therefore necessary to revise the measured data. It is not enough to use only mathematical methods to process data without knowledge of the casting process, because uncertainties and temperature fluctuations have different and often difficult to detect causes. The article deals with sources of temperature uncertainties and fluctuations and methods of extraction of relevant values from measured signals.
\end{abstract}

Keywords: Continuous casting, numerical model, surface temperature measurement, model verification

\section{INTRODUCTION}

Modern casting machines use supporting intelligent measurement and computing systems for process control and diagnostics [1]. One such means is a numerical model of the temperature field of a solidifying steel strand $[2,3]$. From the model, in addition to temperatures, other important quantities can be obtained, such as the thickness of the solid shell, the metallurgical length, the solidification rate etc. Numerical models serve either for research and development purposes, usually in the off-line versions, or as a support model for the operator or the caster control system, in the on-line versions.

The accuracy of the outputs from the numerical model depends on the perfection of the model algorithm, knowledge of steel physical parameters and boundary conditions. Since none of them is ever completely accurate, the numerical model must be fine-tuned and verified, usually by comparing the calculated quantities with those measured in a real process.

There are several quantities that can be used to validate the model, such as shell thickness or metallurgical length measured by various indirect methods including vibration analysis, radioisotope methods or measurement of the solid shell profile after breakout (Figure 1). Most often the models are verified by comparing the calculated and measured surface temperatures of the strand in the secondary and tertiary cooling zones. 


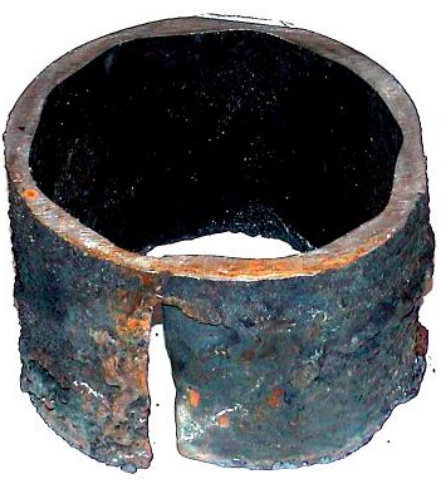

Figure 1 Part of solid shell after breakout

It is known that continuous casting is a dynamic process with changes in parameters and thermal, chemical and mechanical quantities. It is necessary to distinguish between deterministic changes, caused by the operator or control system, and random or quasi-periodic variation caused by spontaneous fluctuations of internal quantities due to disturbances and nonlinearities in the system. Usually, such variations are impossible to include in the model boundary conditions [4]. Then the model calculates only smoothed or average values in time and space with limited accuracy [5].

But, in contrast to the model, all these changes and fluctuations in the process are reflected in the measured surface temperatures. Above that, the measured temperatures are burdened by the uncertainty of the measurement method. Therefore, temperatures must be processed before using them to verify the model. The aim of the research was to develop an empirical-statistical method to obtain relevant values of strand surface temperatures to verify the numerical model. The method and results are demonstrated on real data measured during the selected casting.

\section{NUMERICAL MODELS OF THE STRAND TEMPERATURE FIELD}

Several numerical models of rectangular and round billets and blooms were developed by the authors using either the in-house numerical software based on the finite difference method or the commercial environment ProCAST based on the finite element method. The in-house model was intended for monitoring the strand temperature field, solid shell thickness and metallurgical length in both off-line and on-line versions.

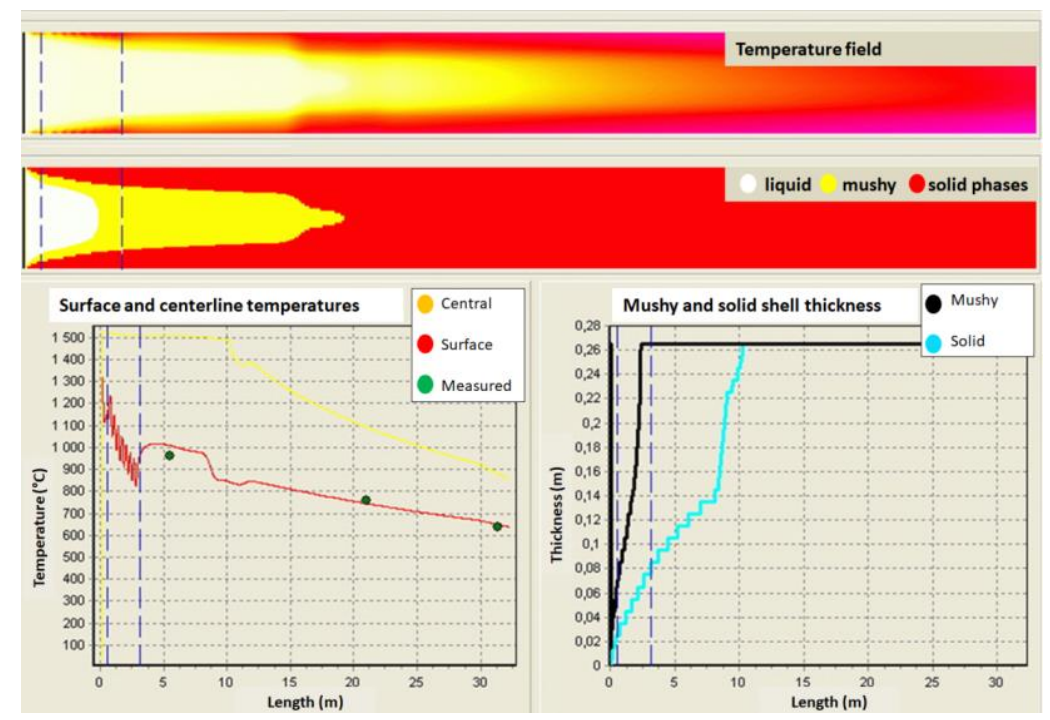

Figure 2 Results from the in-house numerical model

Theoretical background of the thermal model was based on the modified Fourier-Kirchhoff differential equation of the solidifying strand temperature field including the motion of the steel strand.

$$
\frac{\partial i}{\partial \tau}+v_{z} \frac{\partial i}{\partial z}=\frac{\lambda}{\rho} \cdot \nabla^{2} t
$$

where:

$$
\begin{aligned}
& i \text { - specific enthalpy }\left(\mathrm{J} \cdot \mathrm{kg}^{-1}\right), \\
& t \text { - temperature }\left({ }^{\circ} \mathrm{C}\right),
\end{aligned}
$$




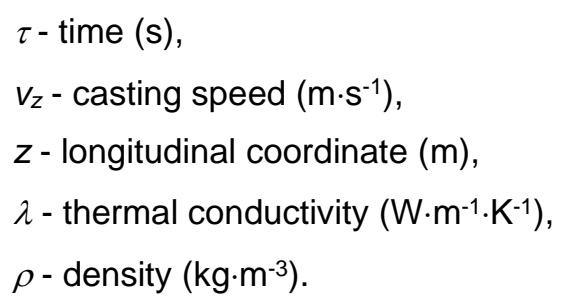

Material properties $\lambda$ and $\rho$ were entered as functions on temperature and steel chemical composition. The latent heat of solidification was included in specific enthalpy as a function of temperature $i=\mathbf{f}(t)$.

An example of results obtained from the in-house on-line model of round bloom of diameter $550 \mathrm{~mm}$ is in Figure 2. The figure shows the current state of the transient after a change in casting speed. In the upper part there are maps of temperature and phase fields. The red curve in the graph bellow them shows the surface temperature of the strand side in dependence on the strand length. Instantaneous values of measured surface temperatures at three individual positions are displayed as green dots. In this case, only three optical pyrometers were installed in the tertiary zone. In other applications, strand surface temperatures in the secondary zone were also measured.

\section{TEMPERATURE MEASUREMENT}

Due to movement of the strand, except for rare applications with tactile thermocouples, mostly contactless optical measurement methods are used. There are basic differences in the method of measurement and data processing according to the purpose of measurement. Unlike measurements for process diagnostics, surface quality and breakout prediction, in the case of measurements for model verification we need absolute and as accurate as possible temperature values. The more measuring points the more accurate is tuning and verification of the model. The most difficult problem is setting the boundary conditions in the secondary cooling area. Ideally, it would be necessary to measure the temperature behind each set of the nozzles whose flow can be adjusted.

The first primary key measure is to avoid optical measurement uncertainties caused by strand surface and atmosphere properties. In previous operational experiments, it has been found that due to the presence of scale and residues of casting powder on the surface, the average temperature measured by an optical pyrometer can be more than $100^{\circ} \mathrm{C}$ lower than the actual temperature. If possible, during the experimental measurement, it is ideal to use a descaling device. It is necessary to use a pyrometer connected to the device for automatic data acquisition. Attention must be paid to the choice of the sensor. It is recommended to measure at wavelengths close to $1 \mu \mathrm{m}$ at which steel has high emissivity and high change in the intensity of radiation depending on the change in temperature. Emissivity of the steel surface depends on temperature, chemical composition and surface properties.

Another limitation is the absorption of radiation in the atmosphere. Due to the presence of water in gaseous and liquid states in the secondary cooling chamber, it is necessary to use a pyrometer with a selective sensor, monochromatic or ideally a ratio pyrometer. In the specific case, Raytek Marathon MR1SA and MR1SB ratio pyrometers were used, which measure at two wavelengths in the range from 0.75 to $1.1 \mu \mathrm{m}$. The field of view was protected by a tube into which compressed air was blown to remove water mist.

As the second basic premise, before the experimental measurement, the casting machine and the casting process must be in perfect condition. In particular, it is necessary to check the wear of the mould and possibly replace with a new one, correct the adjustment of the caster axis, check centring of the submerged entry tube towards the mould and uniform distribution of the casting powder. New nozzles should be installed in the secondary zone. It is necessary to maintain the specific chemical composition and temperature of the steel and to avoid the formation of mixed areas after changing ladles. If all the above measures are not followed, mathematical compensation of measurement errors is very uncertain. 


\section{TEMPERATURE DATA PROCESSING}

The first and most important step is to verify the model under steady casting conditions. Verification of transient states, as shown in Figure 2, is performed mainly in the case of on-line models. When the model is well tuned statically, it usually works properly even during transients.

\subsection{Selection of stable casting time periods and data aggregation}

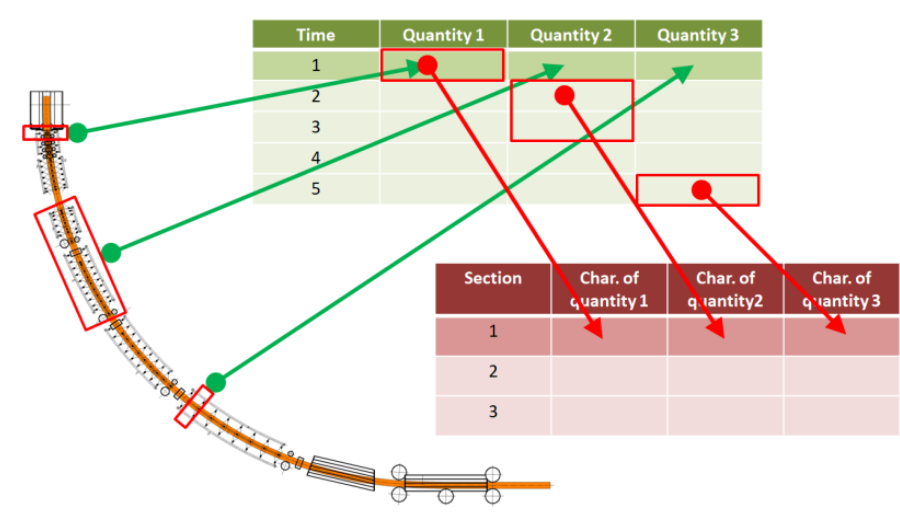

Figure 3 Principle of data aggregation

Periods with constant casting speed and chemical composition of steel are to be selected for steady verification. It is also required to select periods with a constant level and temperature of steel and without changing the secondary cooling setting. Ideally, the steady state should last at least as long as the strand movement time from the steel level in the mould to the last measuring point plus the time it takes to move from the mould to the straightening stool because the rigidity of the strand causes reaction force feedback into the mould. For example, in case of billet caster with

casting speed of $3 \mathrm{~m} \cdot \mathrm{min}^{-1}$ the required time is only about 6 minutes. But in the case of casting the round blooms of diameter $550 \mathrm{~mm}$ at casting speed of $0.3 \mathrm{~m} \cdot \mathrm{min}^{-1}$, the required steady-state time would be more than one hour. Such long steady periods are not available.

If the effect of mechanical bonds is neglected the time is reduced by about half. Then it is advantageous to transform the operational data from the form of time series into a set of so called "independent observations" where each database record represents a history of casting parameters relevant to the virtual transversal slice of the strand during its passage through the casting machine (Figure 3). This significantly reduces the required steady-state period. Instead of time, the data are dependent on the cast length of the strand.

The spacing of the virtual slices is chosen from 1 to $50 \mathrm{~mm}$ depending on the casting speed and the data sampling period. Each casting parameter has defined "the area of action" which can be either point (see quantities 1 and 3 in Figure 3) or interval (quantity 2). Statistical characteristics calculated for each slice and "the area of action" are entered into the new database. The process is called "data aggregation" and special software has been created for this operation. Database records relevant to a sufficiently long part of the strand that was cast under steady state conditions are then selected for further analysis.

\subsection{Data processing of temperature variability}

Even during casting when casting parameters are kept constant, there are stochastic or quasi-periodic changes in measured temperature signals. The minimum length of the selected steady state periods must be at least several times longer than the longest period of temperature oscillation. Further processing of temperature data depends on the causes of temperature changes and is performed in two steps.

The first step is to find and remove short-term temperature drops caused by the spots of a discontinuous layer of oxides and casting powder on the surface as well as local cooling instability. The layer is usually not compact due to thermal shocks under cooling nozzles, vibration and deformation of the strand. Cooling instability is caused by the Leidenfrost effect. Local rapid surface temperature drops relax quickly and do not penetrate deeper, so solidification is not significantly influenced. Therefore, averaging the temperature is not a suitable evaluation method. Temperature drops should be removed from the signal by the moving maximum method. 
The second step is the treatment of slower changes in surface temperature which are related to the process of solidification. These slow oscillations correlate with irregularities in the thickness of the solid shell and the internal structure. They may even manifest mini-ingots formation and variation of central segregation. If the cause of such changes is known, it can be incorporated into the model. The data are then smoothed by a moving average or some more advanced digital filter and the numerical model can be verified dynamically. If the cause is not known, the average temperature is calculated and used for model steady verification.

Figure 4 shows surface temperatures measured in three positions P1, P2 and P3 during the casting of billets with stable casting parameters, the average casting speed being $2.9 \mathrm{~m} \cdot \mathrm{min}^{-1}$. At position P1 just below the mould, there were powder residues and oxides on the surface. The surface at position P2 in the secondary zone was clean without oxides, while at position P3 in the tertiary zone there was a discontinuous layer of scale on the surface. In Figure 5, moving maximum with time window of $2.4 \mathrm{~s}$, which corresponded to the strand length of $116 \mathrm{~mm}$, was applied to the signal measured in the position P3 (the orange curve). Then the values were smoothed by moving average with a time window of $4 \mathrm{~s}(193 \mathrm{~mm})$, see the black curve. For comparison, the original measured signal was also smoothed with the same window (the purple curve).

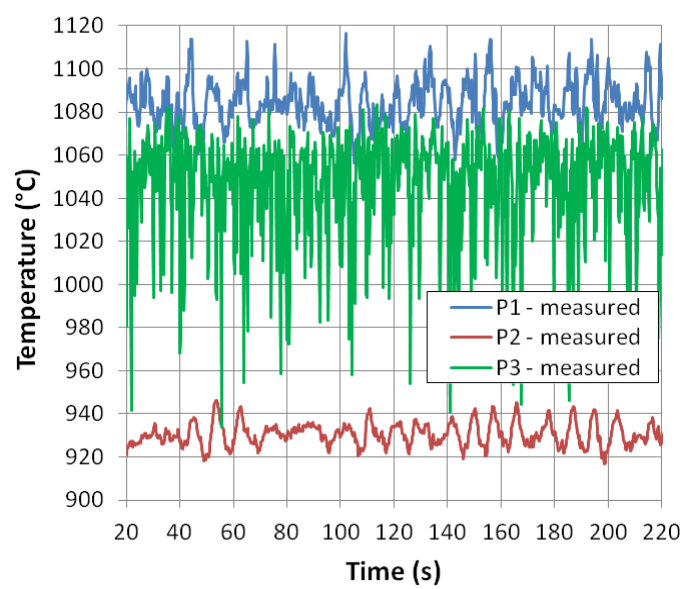

Figure 4 Measured temperatures in positions $\mathrm{P} 1$, $\mathrm{P} 2$ and P3

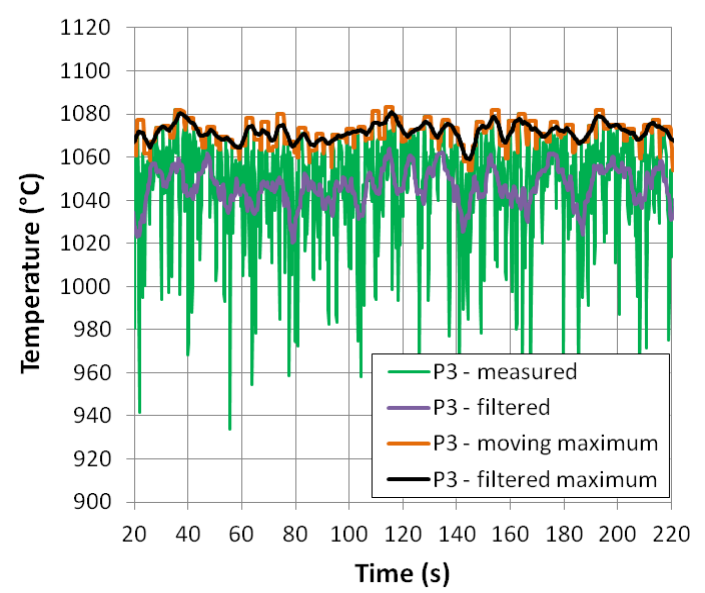

Figure 5 Temperatures in the position P3 before and after data processing

The procedure of steady state selection and data processing is difficult to automate due to the specific conditions of each cast. It is not enough to apply only the mathematical methods without surface observation and knowledge of the process. Stable periods can be identified on the basis of deviation of the actual value from previous smoothed interval. E.g. in this case, the maximum deviation of the casting speed to meet the condition of steadiness was chosen $0.02 \mathrm{~m} \cdot \mathrm{min}^{-1}$.

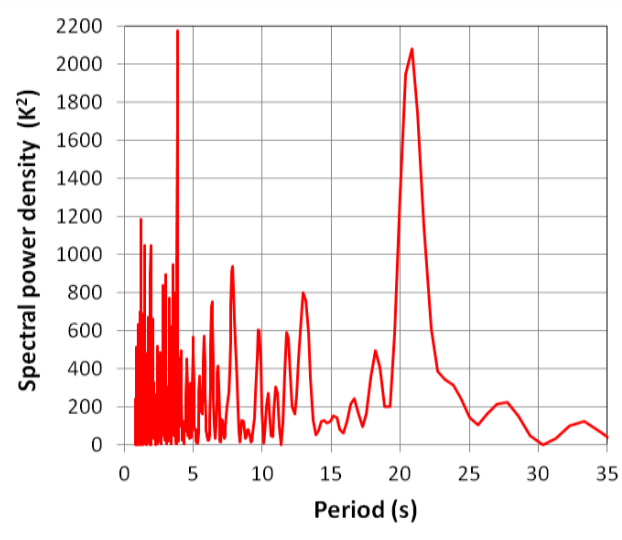

Figure 6 Spectral power density of temperature measured in the position $\mathrm{P} 3$
The choices of the moving maximum window length and the smoothing filter parameters depend on the casting speed and the condition of the strand surface. Frequency analysis of the signal can be helpful. The spectral power density of the measured signal from the measuring point P3 depending on the oscillation period is shown in Figure 6. Fast oscillations with periods shorter than $2 \mathrm{~s}$ (corresponds to the length less than $0.1 \mathrm{~m}$ at casting speed $2.9 \mathrm{~m} \cdot \mathrm{min}^{-1}$ ), which have the character of white noise, should be eliminated from the signal by the moving maximum. Quasi-periodic oscillations with longer periods, e.g. a period of $13 \mathrm{~s}$ (length of $0.6 \mathrm{~m}$ ) or $21 \mathrm{~s}$ (length of $1 \mathrm{~m}$ ), which may be associated with a deterministic behavior, should be preserved in the data. However, if the 
cause of these oscillations is not known, the model can be verified only statically by the mean value of the processed temperature for the entire selected period.

The statistical distribution of temperature values measured at the position P3 during the selected period without data processing was skewed. Therefore, neither the arithmetic mean of $1,047^{\circ} \mathrm{C}$ nor the median $1,053^{\circ} \mathrm{C}$ were usable values. After removing the short-term temperature drops followed by filtration the statistical distribution of data approached the normal symmetrical distribution and the arithmetic mean and median were equally $1,072{ }^{\circ} \mathrm{C}$. In addition, a hidden oscillation appeared with a period of $31 \mathrm{~s}$. Eliminated short-term declines caused by the scale layer and local cooling instability reached up to $120^{\circ} \mathrm{C}$.

\section{CONCLUSION}

Verification of the numerical model of continuously cast strand temperature field according to the measured temperatures of the strand surface requires, above all, the correct choice of the sensor which minimizes the influence of unknown surface emissivity and absorption of radiation in the atmosphere. An optical ratio pyrometer with a protecting tube is the best choice. The casting machine and the casting process must be in perfect condition. Nevertheless, further mathematical processing of measured temperatures is necessary.

Periods with constant casting speed and other key parameters were selected using a special program. This was followed by data transformation using the data aggregation procedure. The temperature variability was then processed in two steps. The statistical distribution of the measured temperature was skewed. Neither the arithmetic mean of $1,047^{\circ} \mathrm{C}$ nor the median $1,053^{\circ} \mathrm{C}$ were usable values for model verification. The method of moving maximum eliminated short-term temperature drops caused by a layer of oxides and residues of casting powder on the surface as well as local cooling instabilities. Eliminated short-term declines reached up to $120^{\circ} \mathrm{C}$. The slower surface temperature oscillations associated with the solidification process were retained in the signal and the data were smoothed by the moving average. After removal the short-term temperature drops followed by filtration, the statistical distribution of temperature approached the normal symmetrical distribution and a new hidden oscillation with a period of $31 \mathrm{~s}$ appeared. The arithmetic mean was equal to the median $1,072{ }^{\circ} \mathrm{C}$ and was used to verify the steady version of the model.

\section{ACKNOWLEDGEMENTS}

This paper originated with the support of grants of the Faculty of Materials Science and Technology, VŠB - TU Ostrava SP2020/34 "Low energy systems and materials in industrial technologies" and SP2020/39 "Specific research in the metallurgical, materials and process engineering".

\section{REFERENCES}

[1] HE, F., HE, D. F., DENG, Z. H., XU, A. J., TIAN, N. Y. Development and application of mould breakout prediction system with online thermal map for steel continuous casting. Ironmaking \& Steelmaking. 2015, vol. 42, no. 3, pp. 194-208.

[2] KAVIČKA, F., DOBROVSKÁ, J., STRÁNSKÝ, K., SEKANINA, B., ŠTĚTINA, J., MASARIK, M. Original industrial application of two numerical models in concasting technology. Advanced Materials Research. 2011, vol. 291-294, pp. 291- 294.

[3] KLIMEŠ, L., ŠTĚTINA, J. Unsteady model-based predictive control of continuous steel casting by means of very fast dynamic solidification model on GPU. Materiali in tehnologije. 2014, vol. 48, no. 4, pp. 525-530.

[4] PYSZKO, R., PŘíHODA, M., FRANĚK, Z., VELIČKA, M., BURDA, J. Non-stationary boundary conditions in thermal models of continuous casting. In METAL 2014: 25th International Conference on Metallurgy and Materials. Ostrava: TANGER, 2016, pp. 54-59.

[5] GUO, L. at all: Mould Heat Transfer in the Continuous Casting of Round Billet. ISIJ International. 2007, vol. 47, no. 8, pp. 1108-1116. 